\title{
PReS-FINAL-2363: Behçet's disease in children: the Great Ormond Street Hospital experience
}

\author{
S Nanthapisal ${ }^{1,2^{*}}$, D Eleftheriou' ${ }^{1}$, Y Hong ${ }^{1}$, N Klein ${ }^{1}$, PA Brogan ${ }^{1}$ \\ From 20th Pediatric Rheumatology European Society (PReS) Congress \\ Ljubljana, Slovenia. 25-29 September 2013
}

\section{Introduction}

Behçet's disease (BD) is rare in childhood and remains challenging in diagnosis and lack of evidence-based data for its treatment. Hence there is an urgent need to understand the scope of the disease in children.

\section{Objectives}

The aim of this study is to describe the clinical spectrum and the therapies used to treat children with Behçet's disease (BD) in children.

\section{Methods}

46 patients (22 male) were identified with a positive family history of BD in 6 cases. Age of onset was 4.87 (0.04-15.71) years with a time to diagnosis of $3.74(0.25$ $13.48)$ years. The main clinical features at presentation were: recurrent oral ulceration (87\%), genital ulceration (20\%), cutaneous symptoms (11\%), fever (30\%), gastrointestinal symptoms (26\%), musculoskeletal (22\%). uveitis (2\%). Recurrent genital ulceration was significantly more common in female patients $(P=0.044)$. The majority of children were treated with colchicine $(74 \%)$ and corticosteroid (41\%). Anti TNF-a treatment was reserved for severe and/or refractory cases (15\%). There was a median of 2 (range $0-12$ ) episodes of oral ulceration per year after the treatment. Interestingly only 10 patients fulfilled The International Study Group (ISG) BD diagnostic criteria.

\section{Results}

46 patients (22 male) were identified with a positive family history of BD in 6 cases. Age of onset was 4.87 (0.04-15.71) years with a time to diagnosis of 3.74 (0.2513.48) years. The main clinical features at presentation were: recurrent oral ulceration (87\%), genital ulceration
(20\%), cutaneous symptoms (11\%), fever (30\%), gastrointestinal symptoms (26\%), musculoskeletal (22\%). uveitis (2\%). Recurrent genital ulceration was significantly more common in female patients $(P=0.044)$. The majority of children were treated with colchicine $(74 \%)$ and corticosteroid (41\%). Anti TNF-a treatment was reserved for severe and/or refractory cases (15\%). There was a median of 2 (range 0-12) episodes of oral ulceration per year after the treatment. Interestingly only 10 patients fulfilled The International Study Group (ISG) BD diagnostic criteria.

\section{Conclusion}

Although most cases were diagnosed in late childhood the first presentation was as early as 1 month old. Delay in diagnosis due to incomplete presentation in certain cases. Oral ulceration was the most common presenting symptom. Uveitis was less frequent than previous series. A range of drugs was used including biologic therapy for severe cases.

\section{Disclosure of interest}

None declared.

\section{Authors' details}

${ }^{1}$ UCL Institute of Child Health, London, UK. ${ }^{2}$ Paediatrics, Thammasat University, Pathumthani, Thailand.

Published: 5 December 2013

doi:10.1186/1546-0096-11-S2-P353

Cite this article as: Nanthapisal et al:: PReS-FINAL-2363: Behçet's disease in children: the Great Ormond Street Hospital experience. Pediatric Rheumatology 2013 11(Suppl 2):P353.

${ }^{1}$ UCL Institute of Child Health, London, UK

Full list of author information is available at the end of the article 